\title{
An Empirical Study on Visual Programming Docker Compose Configurations
}

\author{
Bruno Piedade \\ up201505668@fe.up.pt \\ Faculty of Engineering, \\ University of Porto \\ Porto, Portugal
}

\author{
João Pedro Dias \\ jpmdias@fe.up.pt \\ Faculty of Engineering, \\ University of Porto \\ INESC TEC \\ Porto, Portugal
}

\author{
Filipe F. Correia \\ filipe.correia@fe.up.pt \\ Faculty of Engineering, \\ University of Porto \\ INESC TEC \\ Porto, Portugal
}

\begin{abstract}
Infrastructure-as-Code tools, such as Docker and Docker Compose, play a crucial role in the development and orchestration of cloudnative and at-scale software. However, as IaC relies mostly on the development of text-only specifications, these are prone to misconfigurations and hard to debug. Several works suggest the use of models as a way to abstract their complexity, and some point to the use of visual metaphors. Yet, few empirical studies exist in this domain. We propose a visual programming notation and environment for specifying Docker Compose configurations and proceed to empirically validate its merits when compared with the standard text-only specification. The goal of this work is to produce evidence of the impact that visual approaches may have on the development of $\mathrm{IaC}$. We observe that the use of our solution reduced the development time and error proneness, primarily for configurations definition activities. We also observed a preference for the approach in terms of ease of use, a positive sentiment of its usefulness and intention to use.
\end{abstract}

\section{CCS CONCEPTS}

- Software and its engineering $\rightarrow$ Visual languages; Orchestration languages; Model-driven software engineering.

\section{KEYWORDS}

Visual Programming, Orchestration, Docker, Docker Compose

ACM Reference Format:

Bruno Piedade, João Pedro Dias, and Filipe F. Correia. 2020. An Empirical Study on Visual Programming Docker Compose Configurations. In ACM/IEEE 23rd International Conference on Model Driven Engineering Languages and Systems (MODELS '20 Companion), October 18-23, 2020, Virtual Event, Canada. ACM, New York, NY, USA, 10 pages. https://doi.org/10.1145/ 3417990.3420194

\section{INTRODUCTION}

As the user-base and complexity of applications grow the infrastructure upon which they are built will often also need to grow. As a

Permission to make digital or hard copies of all or part of this work for personal or classroom use is granted without fee provided that copies are not made or distributed for profit or commercial advantage and that copies bear this notice and the full citation on the first page. Copyrights for components of this work owned by others than the author(s) must be honored. Abstracting with credit is permitted. To copy otherwise, or republish, to post on servers or to redistribute to lists, requires prior specific permission and/or a fee. Request permissions from permissions@acm.org.

MODELS '20 Companion, October 18-23, 2020, Virtual Event, Canada

(C) 2020 Copyright held by the owner/author(s). Publication rights licensed to ACM ACM ISBN 978-1-4503-8135-2/20/10 ..\$15.00

https://doi.org/10.1145/3417990.3420194 result, several challenges appear in software operations, namely in the management and scaling of infrastructure, which is, ever more commonly, cloud-based. Containers and Infrastructure as Code (IaC) have been playing a key role in how software developers tackle this increase in infrastructure complexity.

Containers have been enablers of microservice architectures [1], allowing the creation of self-contained software units, consisting of, for example, services and applications, in a more lightweight way when compared to virtual machines $[6,17]$. One of the most known container creation and management solutions is Docker (and Docker Compose), which currently plays a massive role in this context [10].

Infrastructure as Code $(\mathrm{IaC})$ is the practice of managing infrastructure through configuration files as part of the code-base [5, 18]. Although initially designed for configuring and managing baremetal infrastructure (e.g., Chef, CFEngine and Puppet [11]), this practice expanded for managing and provisioning infrastructure resources in the cloud $[16,18]$.

Docker Compose is a tool for orchestrating multiple services using Docker. It supports defining orchestration configurations through YAML files, which specify the services that constitute the application, the corresponding images, and how they are related to each other as well as volumes for data persistence and networks for the connections between services. The services can then be run, conventionally through the command-line interface (CLI), resulting in the creation or execution of the declared resources, including the container or set of containers for each of the declared services.

Although this process may be fairly straight-forward for the setup of low complexity orchestration configurations, the textual nature of the configuration files may present some challenges as the complexity increases, be it in number or in the heterogeneity of services. In such cases, it is evident that understanding dependencies between services becomes difficult as definitions begin to get scattered within the file. Furthermore, some advanced aspects of the configuration, such as port mapping and volume management, might be overwhelming and confusing for inexperienced users.

Some of the research on solutions for dealing with high-complex text-based systems have focused around abstraction models that hide low-level complexity had to succeed at a certain degree. For example, in manufacturing industries the configuration of programmable logic controllers (PLC) via solutions such ladder and sequential function charts as a complement to textual representations [19] and in software engineering the usage of visual notations 
such as the Unified Modeling Language (UML). More recent applications can be found for educational purposes and in the area of Internet of Things (IoT) [4].

There are already numerous examples of visual approaches found in the operations field for multiple purposes and tasks, for instance, the management of cloud and container resources, including some which focus on Docker technologies [9]. In this sense, there is evidence supporting the viability and usefulness of such an approach in broader cases, including Docker Compose. Furthermore, there is currently a high concern with misconfigurations resulting from IaC scripts following the widespread of this process to automate infrastructure provisioning and management [13].

In this paper, we delve into the benefits of a visual programming approach applied to service orchestration based on Docker Compose by evolving and expanding the efforts already made in this field. We present a complete visual programming approach including the definition of the associated graphical elements and visual notations for the artefacts and relations inherent to Docker Compose, implementing such approach in a prototype which works as a proof-of-concept. We then proceed to validate our approach with a series of experiments with developers, assessing their performance when compared to current solutions.

This paper is structured as follows: Section 2 presents an overview of related work and open research challenges and Section 3 gives some insights on the Docker Composer prototype. Section 4 details our experimental setup and Section 5 presents and discuss the obtained results. Finally, some closing remarks are given on Section 6 , pointing limitations and future work directions.

\section{RELATED WORK}

Two topics stand out given the goals of this work-how model-driven engineering techniques have been used to address issues within the domain of cloud infrastructure, and the extent to which visual programming approaches have been used for service orchestration.

\subsection{Model-driven Cloud Infrastructure}

Cloud computing has been the target of numerous model-driven approaches following its massive proliferation and evolution, which promoted the need for new and better solutions. Such approaches have surfaced to improve the way resources are managed in cloud environments by working at a higher abstraction level provided by high-level models as an alternative or complement to conventional low-level configurations.

Ardagna et al. [2] proposed the initial vision of MODAClouds, a framework to tackle the challenges inherent to the lack of interoperability between cloud service providers and resulting lock-in to some provider. The goal was achieving a service-agnostic approach fit for running applications in cross-provider multi-clouds settings, appropriate for strict high availability and flexibility non-functional and business requirements. To this end, a model-driven approach was proposed, allowing the design of software system model artefacts at distinct abstraction levels which are ultimately transformed into code and automatically deployed in the target cloud platforms.

Sandobalin et al. $[14,15]$ proposed ARGON as a DevOps support tool leveraging IaC practices and its benefits for infrastructure provisioning while simultaneously minimizing its drawbacks through a model-driven approach. The project defines a DSL to model infrastructure independently of the provider, later used to generate the target tool script. It builds upon previous efforts (such as the mentioned [3]) but innovates by operating on top of existing Configuration Management Tools (CMTs) such as Chef and Puppet, creating a toolchain in which pipelines can be configured to update on change automatically. Afterwards, the authors proposed an extension to the DSL to support a more comprehensive set of resources and compared the practical effectiveness of ARGON in comparison to the well-established scripting-based Ansible ${ }^{1}$ with promising results.

Model-driven approaches have also been applied directly in the context surrounding Docker containers. Paraiso et al. [12] explored the applicability of a model-driven approach to address some of the drawbacks of these technologies, especially in regards to, among others, deployment processes and maintenance in production environments. The result was a modelling tool built in a 3 component architecture: (1) Docker Model, a model for containers and their dependencies, (2) Executing Environment, the target infrastructure hosting the containers and (3) the Connector working as the bridge and providing synchronism mechanics between the previous two. An Eclipse-based prototype was developed named Docker Designer, featuring a GUI to work with these technologies.

\subsection{Visual Programming Service Orchestration}

While there is a considerable amount of visual approaches related to multi-service systems, most of them focus on defining behaviours for monitoring but not for defining the service orchestration per se. The following works are some that we considered relevant in the context of defining, understanding and evolving orchestration and infrastructure specifications. Since research works are scarce, we have also considered grey literature.

Kitematic ${ }^{2}$ provides a form-based GUI for managing Docker containers. Besides actions such as creating, restarting, and deleting a container, it supports the management of images, volumes, and networks. Furthermore, it includes automatic port-mapping and integration with Docker Hub for direct image searching. It currently stands as one of the most common visual tools for Docker since it is part of the official Docker Toolbox.

Dockstation $^{3}$ provides a GUI for handling Docker containers in local and remote environments and is aimed primarily for development purposes. The "Project" feature represents a Docker Compose configuration, specified in a docker-compose.yml file. After the creation of a new project, during which an existing docker-compose.yml file can optionally be imported, and the developer can (1) visualize the overall services scheme featuring a visual representation of each service as well as their relationships and dependencies in a dataflow-like diagram and (2) edit each service via forms for some of its properties (i.e., environment variables, volumes, and ports). Whenever the user edits some property, the changes are then reflected on the associated docker-compose.yml accessible in the "Editor" tab and vice-versa.

\footnotetext{
${ }^{1}$ Ansible, available at https://www.ansible.com/

${ }^{2}$ Kitematic, available at https://kitematic.com/

${ }^{3}$ Dockstation, available at https://dockstation.io/
} 
Admiral $^{4}$ is as a web-based GUI for container management and provisioning, with a focus on deployment and production maintenance. It supports multi-container specifications by the definition of "Templates" that include four main components: containers, volumes, networks, and closures. Each is created and configured via a form-based interface. It is then possible to visually connect each container or closure with a network or volume by dragging the mouse from the source component to the target element.

Weerasiri et al. [20] present CloudMap as a solution to "simplifying the way DevOps can navigate and understand cloud resource configurations, as well as monitor and control such resources". It aims to provide a centralized system for cloud management and monitoring by integrating crucial features which are usually found across multiple independent technologies. In particular, the resulting visual notation presented in a map-like fashion. The resulting visual notation is inspired by the dataflow programming metaphor, representing resources and their connections. The reasoning behind this choice and a more detailed description of what each visual element represents is supplied by the authors in the source work [20].

Other authors present other visual alternatives, such as CloudCity, which explores the benefits of model-driven engineering techniques applied to cloud management through a 3D city-like visual metaphor [7, 8].

\subsection{Discussion}

Regarding current model-driven solutions for cloud, both modelto-model and model-to-code have been proposed. However, they mostly focus on resolving the vendor lock-in issue and facilitating work in multi-cloud settings. However, Sandobalin et al. [14, 15] already propose the development of $\mathrm{IaC}$ with high abstraction level programming, being this close to our work focus.

While there is a considerable number of works and tools that focus on abstracting the complexity of creating, managing and evolving IaC-based systems, most of these rely on form-based or textual methods, not featuring a truly visual method for this purpose. Those that do (Dockstation and Admiral) are either under development or incomplete in working as visual orchestrators, often overly relying on forms and lacking visual notations for certain artefacts and therefore do not offer a completely visual approach. Furthermore, there is a lack of empirical evidence that such approaches improve the development process of such systems.

There is a lack of exploration of distinct visual metaphors, with most adopting a blocks-and-links (e.g., dataflow-like) approach Notwithstanding the adequacy of such a metaphor, we believe there may be potential in exploring others which may be easier to understand, especially for inexperienced developers.

\section{THE DOCKER COMPOSER TOOL}

To allow users to define Docker Compose configurations visually through the manipulation of an interactive and visual model of an orchestration specification in opposition to the conventional textbased fashion, a visual programming environment featuring the designed complete visual approach for visualizing and orchestrating services with Docker Compose and Docker technologies was developed, named Docker Composer. The prototype has a twofold

\footnotetext{
${ }^{4}$ Admiral, available at https://github.com/vmware/admiral
}

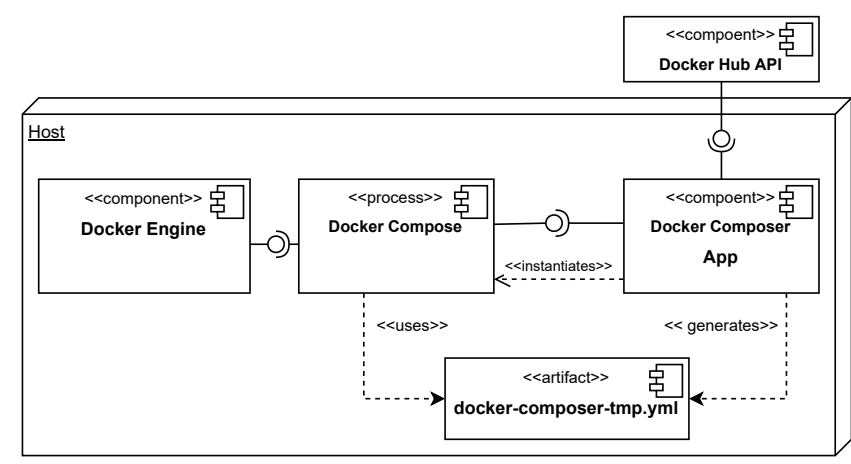

Figure 1: Deployment diagram of the prototype.

goal: (1) to serve as a reference implementation to demonstrate the approach in practice and (2) use the prototype as a means to empirically validate the visual approach.

Fig. 1 displays a deployment diagram showcasing a high-level view of the prototype and its interactions within and outside the host environment. Within the host environment, the prototype generates Docker Compose YAML artifacts and creates processes running shell instances for the execution of Docker Compose CLI commands which in turn communicate with the Docker Engine. Outside of the host environment, the prototype performs requests to the remote Docker Hub's public API in order to receive information about the images hosted on this service.

Fig. 2 presents the prototype's main view. The view is split into five panels (as labeled in Fig. 2), namely:

Toolbar Located at the top of the screen. Includes to the left an area dedicated to controlling status LED which lights up different colors according to the state of the running orchestration specification and a set of buttons to control the services (such as starting and stopping) and on the right buttons for file management. In the settings menu, you can set the working directory and adjust some output preferences when exporting files.

Image Palette Allows searching for images hosted on Docker Hub and the addition of new services by clicking and dragging the target image and dropping it in the graph editor area.

Graph Area Split between the toolbar at the top and the canvas below. The toolbar is used to perform actions such as adjusting the zoom level and clearing the graph editor. The canvas displays an interactive visual map of the orchestration specification containing the various artifacts that comprise it and their relationships.

Properties Editor Useful to access and edit the various properties of the currently selected object (artifact or connection) in the graph editor.

Terminal Displays the output produced by the services (containers) once created and started. It contains a "General" tab with the combined output of all services and additional logs (i.e., Docker Compose logs) and individual tabs for the output of services that comprise the orchestration specification.

As an example, to more clearly compare and demonstrate the differences of representation between the conventional text-based 


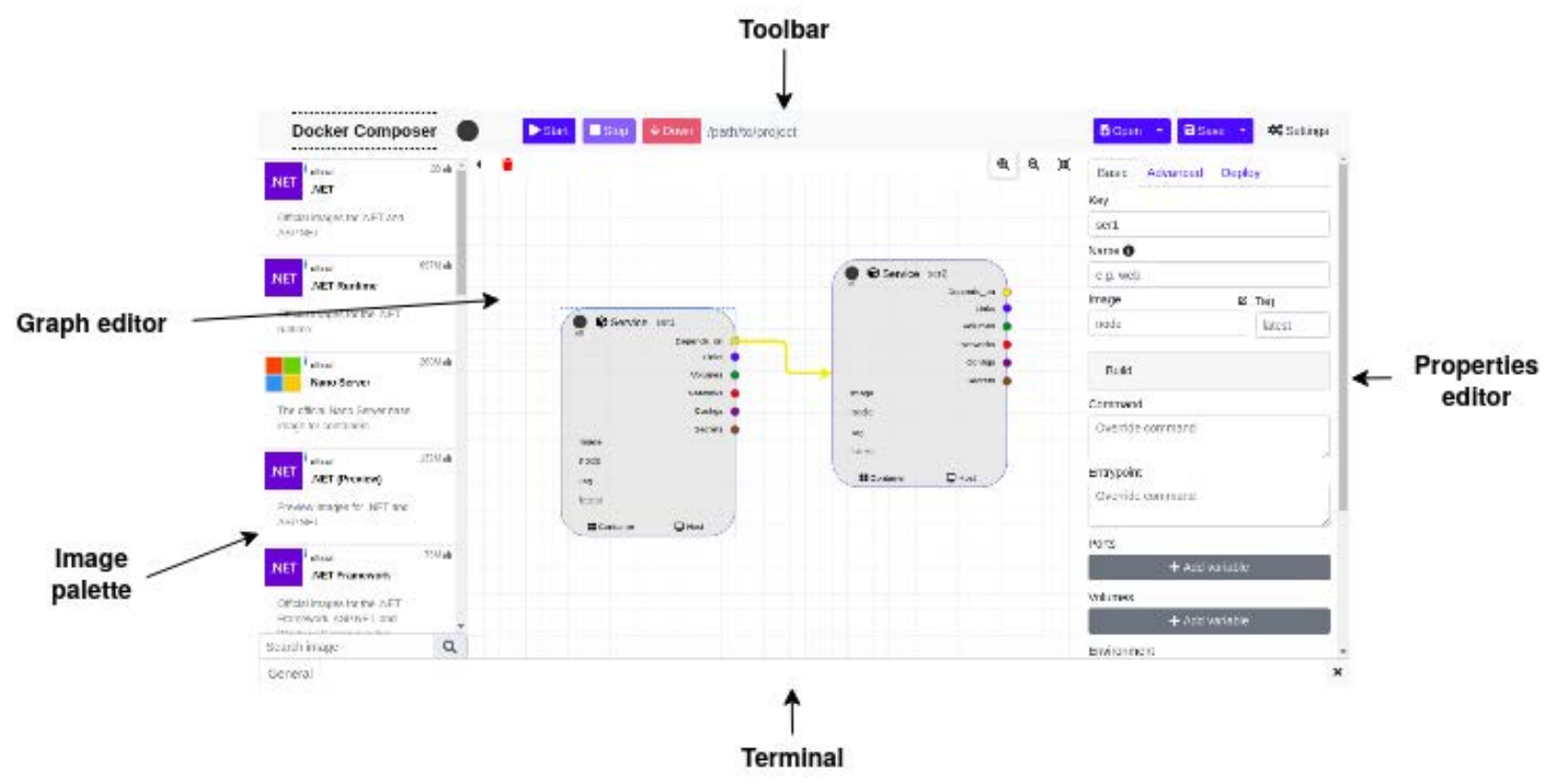

Figure 2: Layout of the prototype's main view, showcasing the graph editor (drawing canvas), main control toolbar, image palette (from Docker Hub) and the properties editor (corresponding to one of the services in the canvas).

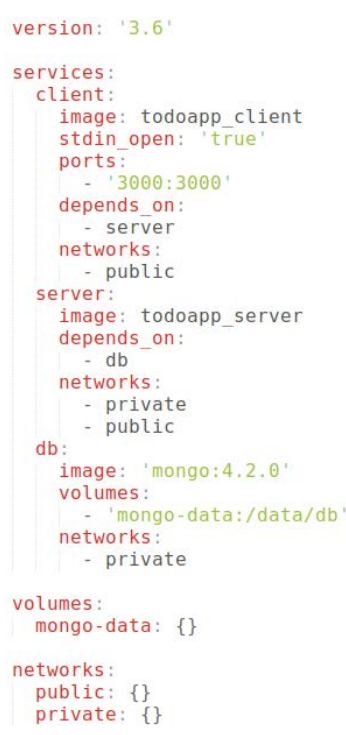

(a) Docker Compose.

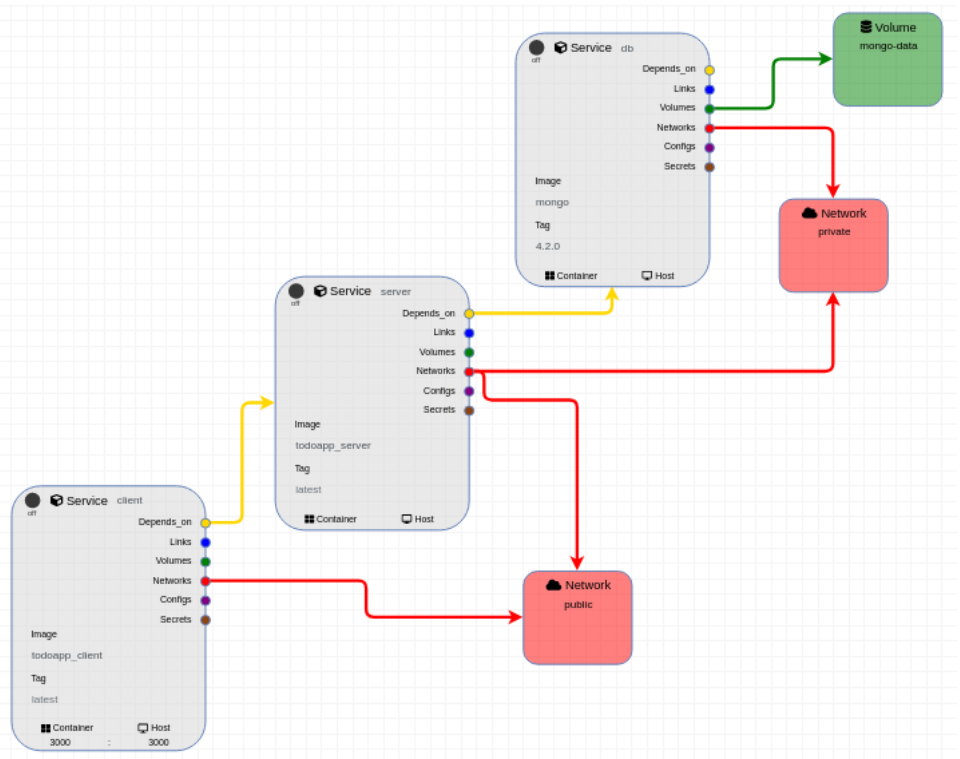

(b) Docker Composer.

Figure 3: Concrete example of a Docker compose specification. (a) presents the default textual representation of a docker-compose. yml file, and (b) a visual representation of the same configuration using Docker Composer.

approach and the designed visual approach, we present Fig. 3. The presented orchestration specification follows a server-client architecture comprised of three services: a web frontend service (client), a backend web service (server), and MongoDB database service (db). We also include two custom networks, named private and public, to isolate the backend from the frontend as well as a named volume, called mongo-data, for data persistence.

Fig. 3 showcases a side-by-side comparison between the textual representation (a) and the equivalent visual representation (b). While it may not be immediately clear, both representations 
Table 1: Comparison of the supported visual notations for Docker Compose parts-i.e., services, volumes, networks, configs and secrets-by different solutions.

\begin{tabular}{lccccc}
\hline Name & Services & Volum. & Net. & Configs & Secrets \\
\hline Docker Composer & $\checkmark$ & $\checkmark$ & $\checkmark$ & $\checkmark$ & $\checkmark$ \\
Admiral & $\checkmark$ & $\checkmark$ & $\checkmark$ & $x$ & $x$ \\
Dockstation & $\checkmark$ & $x$ & $x$ & $x$ & $x$ \\
\hline
\end{tabular}

convey the same information. While the visual approach makes the artifacts themselves and their connections more evident, some information becomes obscured, namely, the remaining properties (e.g., stdin_open on the client service). To mitigate this limitation of the visual approach, it is possible to hover on top of any artifact to visualize the full-textual output for that artifact.

The designed visual approach differs from other state-of-the-art solutions as none offers a complete visual notation for the artifacts and dependencies considered in Docker Compose. This was the primary goal during the conceptual stage and throughout the development process itself. Table 1 displays the comparison of the visual notations featured in Docker Composer and those of the more closely related state-of-the-art tools-Dockstation and Admiral.

The prototype also provides some static validations while editing a stack. Examples include duplicate key detection and invalid property value formats (e.g., durations and memory sizes). These are conveyed to the users through warning icons which appear near the visual representation of artifacts which provide more info when hovered. Additionally, the connections between boxes are typed, thus erroneous connections (e.g., connecting a Networks gate to a Service block) are not allowed.

\section{EXPERIMENTAL SETUP}

The empirical study focuses on the evaluation of three activities in software engineering-analyzing, debugging, and implementing-in the context of Docker Compose configurations. A task was prepared for each activity, and performance-based metrics were collected, namely: effectiveness, completion times and execution attempts.

\subsection{Participants}

A total of 16 students from the MSc in Informatics and Computing Engineering participated in the experiment. All had prior experience with Docker and Docker Compose due to their academic path and were randomly evenly distributed between two groups, corresponding to the treatments: control (CG) and experimental (EG). Both groups were asked to solve the same set of tasks. The participants of the CG had access to a text editor to edit the orchestration specification and to a command-line shell to access the conventional toolchain. The participants of the EG had access to the experimental prototype to manage the orchestration specification as well as a command-line shell to execute additional commands if required (Docker related or not). In addition, both groups had complete access to the official Docker and Docker Compose documentation as well as any other resources on the internet.

\subsection{Environment}

The experimental sessions were conducted remotely. We opted for a remote workstation, set up in advance with the required software and materials, which was later made available to the participants. These resources included a browser (to access the experimental guidelines and surveys), a text editor set up in the appropriate directory, a command-line shell set up in the appropriate directory for both groups and the prototype tool for the EG.

\subsection{Task Definition}

As previously stated, the goal was to evaluate the behavior of the tool for three basic activities: analyzing, debugging, and implementing an orchestration specification. This effort was translated into 4 tasks each featuring a corresponding scenario. In Task 1 (T1) a functioning Docker Compose configuration was provided and the goal was to analyze its structure and understand the overall behavior. In Task 2 (T2) a buggy configuration was provided and the goal was to debug and fix the faulty behavior. Task 3 (T3) focused on implementing and was divided into T3.1-build a simple configuration from the ground up (implementation)-and T3.2-modify the configuration to use secrets (increment).

To ensure a balance between scale, complexity, realism, and expected time to completion in the tasks, a brief study was conducted to identify the scale (in terms of the number of services per configuration) distribution of Docker Compose configurations in real-life projects, using GitHub as a data source. We concluded that most projects (approximately 75\%) had low complexity (i.e., 1 to 2 services).

\subsection{Procedure}

A full session took between 50 minutes to 2 hours per participant. Each session was conducted individually with the researcher overseeing and observing the full procedure. Communication was done via remote voice call. The participants were encouraged to think aloud throughout the session so that the researcher could more clearly understand and follow along with their rationale. This strategy was also useful in identifying potentially unforeseen issues with the experiment's design.

Once the connection to the remote workstation was established, the participant had access to the instructions for the full procedure available in the remote environment, which we make available as a replication package ${ }^{5}$. The procedure was organized in the following sections:

Background Survey This survey contained a set of questions to assess the current degree of experience with technologies which we had foreseen to potentially be confounding factors.

Tutorial Before solving the actual tasks, the participants had to follow a simple tutorial reviewing some basics of Docker Compose. This was mostly targeted to the EG so that they had some prior hands-on experience with the prototype. Nonetheless, to maintain consistency between both groups, participants in the CG also had to achieve the same goal with the conventional toolchain.

\footnotetext{
${ }^{5}$ A replication package to facilitate and encourage the independent replication of this experimental design is available at https://zenodo.org/badge/latestdoi/281461421.
} 
Experimental Tasks Participants were instructed to solve a set of four orchestration-related tasks. To maintain the total duration reasonable, time limits were set for each task. Participants were asked to advance to the next task whenever this time limit was exceeded.

Post-experimental Survey Participants were asked to fill a survey to assess their experience and evaluate the experience of working with the tools. The survey in the EG differed from the control since it included an additional set of questions to specifically evaluate the solution prototype.

\section{RESULTS AND DISCUSSION}

The data collected was mainly quantitative and was used for hypothesis testing-the Mann-Whitney $U$ (MW-U) and McNemar tests against our variables of interest. The notation used represents $H_{0}$ as the null hypothesis and $H_{1}$ as the alternative hypothesis, $\mathrm{u}$ for the U statistic of MW-U tests, and $\rho$ as the probability of rejecting $H_{0}$. We also denote $\sigma$ as the standard deviation and $\bar{x}$ as the mean.

\subsection{Background and Tutorial}

The background survey gathered information about confounding factors, to support ensuring that the groups are balanced in experience and skills. Questions are defined as Likert items and numeric values and inquire if the participants consider themselves experienced with (1) visual programming, (2) orchestration frameworks and tools, (3) Docker and (4) Docker Compose-configuration of volumes, networks, configs and secrets.

We have run MW-U and McNemar hypothesis tests to check if there was any significant difference between groups, and they revealed that the participants were indeed balanced across both groups. To further ensure the groups were under equivalent conditions, they have run a simple tutorial in the respective toolchain that they were requested to use during the tasks.

\subsection{Experimental Tasks}

During the task we measured the task completion, work context times, task times, execution attempts and context switches, as analysed next.

5.2.1 Task Completion. We consider effectiveness as the ratio between successfully completed tasks and the total number of tasks. A task is successfully complete only if the subject finished within the allotted time limit and the solution was correct.

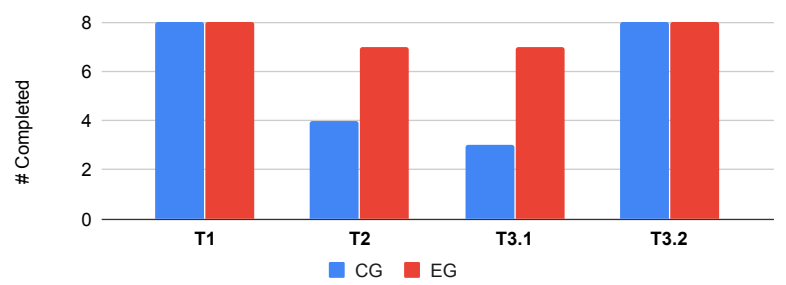

Figure 4: Distribution of the total completed tasks per group.

Fig. 4 displays the distribution of completed tasks by group. While all participants completed T1 and T3.2, there is a clear difference in T2 and T3.1. While most of the participants in the EG
Table 2: Summary of the global time registered per activity for the sum of time taken in all tasks, with the mean and standard deviation for each group.

\begin{tabular}{lccccccc} 
& \multicolumn{4}{c}{ CG } & & \multicolumn{3}{c}{ EG } \\
\cline { 2 - 4 } \cline { 6 - 8 } Context & $\sum$ & $\bar{x}$ & $\sigma$ & & $\sum$ & $\bar{x}$ & $\sigma$ \\
\hline Script & $2: 06: 04$ & $15: 46$ & $06: 10$ & & $1: 29: 40$ & $11: 13$ & $03: 52$ \\
Composer & - & - & - & & $3: 50: 08$ & $28: 46$ & $10: 29$ \\
Docs & $1: 51: 36$ & $13: 57$ & $06: 25$ & & $0: 18: 59$ & $02: 22$ & $02: 15$ \\
Browser & $0: 41: 44$ & $05: 13$ & $04: 02$ & & $0: 11: 36$ & $01: 27$ & $01: 53$ \\
Editor & $2: 52: 17$ & $21: 32$ & $03: 57$ & & $0: 04: 51$ & $00: 36$ & $00: 29$ \\
Terminal & $1: 53: 03$ & $14: 08$ & $02: 38$ & & $0: 02: 10$ & $00: 16$ & $00: 31$ \\
Stack Man. & $3: 34: 01$ & $26: 45$ & $07: 16$ & & $3: 54: 59$ & $29: 22$ & $10: 47$ \\
\hline
\end{tabular}

completed the experimental tasks, only approximately half of the participants in the $\mathbf{C G}$ were able to complete them. The participants in both groups who were unable to complete the tasks was due to the imposed time-constrains on solving them. No case was registered in which the solution was incorrect. We can conclude that fewer participants in the CG finished the task T2 and T3.1. This, in turn, has an impact on the metrics considered for the remainder of this analysis since the registered times were capped up to the moment when the time limit was exceeded. If the time limit was not set the differences may have been even sharper. However, this was a necessary sacrifice to keep the overall time reasonable and manageable.

5.2.2 Work Context Times. The times spent on each work context allow us to better understand the behavior of the participants.

Table 2 overviews the global times per context. We shall first look into those that are directly comparable between the two groupsScript, Docs (documentation) and Browser.

Table 3: Result of the Mann-Whitney U equality test for the sum of time spent on three contexts.

\begin{tabular}{lcrr} 
Context & $H_{1}$ & $\mathrm{u}$ & \multicolumn{1}{c}{$\rho$} \\
\hline Script & $>$ & 17 & 0.065 \\
Docs & $>$ & 2 & $<0.001$ \\
Browser & $>$ & 9 & 0.007 \\
\hline
\end{tabular}

Considering the alternative hypothesis that the time spent in the Docs and Browser contexts is higher for the CG, the results shown in Table 3 confirm that, indeed, the CG spent significantly longer than the EG in these contexts. We can also see that there is not a significant difference between the groups in the time spent on the Script context for reading the script.

When considered individually, it is difficult to draw any other useful information from the remainder of the variables as these were either exclusive to some group (i.e., Composer for the EG) or partly replaced the purpose of one another across both groups. However, we can consider the sum of time spent on editor and terminal $(\mathrm{E}+\mathrm{T})$ in the CG to be roughly equivalent to the sum of time spent on the textual editor-which mostly equates to the time spent accessing other textual materials such as configuration files which were used 
in the tasks-and on the solution prototype Composer $(\mathrm{E}+\mathrm{C})$ in the EG. No participant in the EG used the terminal to execute any other Docker or Docker Compose CLI commands besides those that were available in the prototype. We refer to this composite context focused on the management of the stacks as Stack Management, and show it as the last line in Table 2. The time difference shown in this line does not appear to be very high. Testing the hypothesis (CG > EG using the MW-U test rendered $u=29$ and $\rho=0.399$. These results do indeed not show that the participants in the EG have spent significantly less time managing the services than those of the CG.

Therefore, it seems reasonable to conclude that the biggest impact on the overall duration was the time spent consuming documentation resources. This difference is in-line with the expectation that a VPL in this context promotes an exploratory approach in which the solution space is constrained by the options that are explicitly made available through the user interface, and users are able to converge to solutions by searching the available options provided by our prototype.

5.2.3 Task Times. Analyzing the times per context provides detailed insight into the behavior of the participants. We can, however, also look at the time spent globally (i.e., the total sum of time spent on each activity) to assess the overall speed.

Table 4: Summary of the completion times for each task across groups.

\begin{tabular}{lccccccccc} 
& \multicolumn{2}{c}{ CG } & & \multicolumn{2}{c}{ EG } & & \multicolumn{3}{c}{ MW-U } \\
\cline { 2 - 3 } \cline { 8 - 9 } Task & $\bar{x}$ & $\sigma$ & & $\bar{x}$ & $\sigma$ & & $H_{1}$ & u & $\rho$ \\
\hline T1 & $0: 13: 05$ & $0: 05: 51$ & & $0: 12: 12$ & $0: 05: 19$ & & $>$ & 31 & 0.480 \\
T2 & $0: 22: 59$ & $0: 04: 55$ & & $0: 14: 41$ & $0: 05: 59$ & & $>$ & 11 & 0.014 \\
T3.1 & $0: 24: 56$ & $0: 07: 04$ & & $0: 13: 47$ & $0: 06: 57$ & & $>$ & 3 & 0.001 \\
T3.2 & $0: 09: 35$ & $0: 04: 26$ & & $0: 04: 01$ & $0: 01: 56$ & & $>$ & 6 & 0.002 \\
\hline
\end{tabular}

Table 4 summarizes the results obtained for the times of each task along with the results of the MW-U significance test performed to compare both. By considering this data and the expected alternative hypothesis which states that the participants in the EG would finish tasks faster than those of the CG (i.e., CG > EG) for all tasks, the results demonstrate that EG did indeed finish task T2, T3.1 and T3.2 significantly faster than the CG. These tasks evaluated debugging, implementing, and updating activities. Particularly, it is interesting to note the significant difference in T3.2. The scenario in this task required the participants to use a somewhat uncommon feature of Docker Compose-secrets-with which most did in fact not have any prior experience. In practice, the workflow to utilize this feature in the prototype was very similar to that of other artifacts, such as volumes and networks. These results support that the prototype was sufficiently intuitive for participants to learn how to use this new feature, after having some experience with it, simply by following a similar rationale and without the need to consult additional documentation.

The prototype was successful in reducing the overall time required to develop and debug orchestration specifications. While there was not a meaningful improvement for task T1 (in which participants had the goal of analyzing an orchestration specification), overall, the prototype managed to reduce the time for the remainder of tasks. Some of the questions in T1 required a deeper knowledge of concepts that were not immediately conveyed by the prototype. We suspect that, taking into account that although the participants in EG already had some hands-on experience with the prototype during the tutorial, T1 being the first task in conjunction with the more complex questions contributed towards the longer duration since the participants spent some time exploring the features of the prototype searching for answers.

5.2.4 Execution Attempts. In addition to the task times, the execution attempts were also registered for each task, that is, the number of times a participant tried to run the orchestration specification (i.e., run the command docker-compose up).

Table 5: Summary of the execution attempts for each task across both groups.

\begin{tabular}{|c|c|c|c|c|c|c|c|}
\hline \multirow[b]{2}{*}{ Task } & \multicolumn{2}{|c|}{ CG } & \multicolumn{2}{|c|}{ EG } & \multicolumn{3}{|c|}{ MW-U } \\
\hline & $\bar{x}$ & $\sigma$ & $\bar{x}$ & $\sigma$ & $H_{1}$ & $\mathrm{u}$ & $\rho$ \\
\hline T1 & 0.38 & 0.518 & 0.50 & 0.535 & $>$ & 28.0 & 0.500 \\
\hline $\mathrm{T} 2$ & 7.00 & 3.928 & 5.63 & 2.560 & $>$ & 21.5 & 0.134 \\
\hline T3.1 & 10.13 & 4.357 & 5.25 & 4.097 & $>$ & 11.5 & 0.014 \\
\hline T3.2 & $3: 50$ & 1.690 & 1.75 & 0.463 & $>$ & 13.0 & 0.016 \\
\hline
\end{tabular}

Table 5 displays the results for execution attempts. Considering this data and the expected alternative hypothesis which states that the EG would need fewer execution attempts than the CG (i.e., CG > EG) for all tasks, the results demonstrate that EG did indeed require significantly fewer execution attempts for T3.1 and T3.2.

These results are in line with the time difference established above. Overall, the participants in the EG were more efficient and did not spend as much time restarting the services. This behavior was also expected as in practice many execution attempts in the CG resulted from syntax errors. The prototype avoided most syntax errors simply due to the more strict form inputs (with stronger validation) and subsequent automatic code generation which was free of errors. We believe that this was the biggest factor to contribute to the non-significant difference in $\mathrm{T} 2$ since in this task a partially working orchestration specification was provided and few changes were required.

5.2.5 Context Switches. In addition to the work context times, the context switches were also recorded, that is, the number of times the participant accessed each of the contexts. To keep this metric uniform across the participants we will consider the context switches per minute $(\mathrm{s} / \mathrm{m})$ as opposed to the total count of context switches. This metric is useful in evaluating the degree of focus of participants which has a substantial impact on its usability. We argue that, in general, a higher number of context switches translates into a less optimized experience since a user has to shift their attention more frequently, therefore being more distracting.

We will analyze the global context switches during the full session, that is, the total sum of the switches between all contexts for all tasks.

To assess if the participants in the EG performed fewer context switches than those of the CG, a MW-U test was performed (Table 6). 
Table 6: Results of MW-U test for global context changes.

\begin{tabular}{|c|c|c|c|c|c|c|c|}
\hline \multirow[b]{2}{*}{$\mathrm{s} / \mathrm{m}$} & \multicolumn{2}{|c|}{$\mathrm{CG}$} & \multicolumn{2}{|c|}{$\mathrm{EG}$} & \multicolumn{3}{|c|}{ MW-U } \\
\hline & $\bar{x}$ & $\sigma$ & $\bar{x}$ & $\sigma$ & $H_{1}$ & $\mathrm{u}$ & $\rho$ \\
\hline Global & 4.628 & 0.912 & 3.479 & 0.929 & $<$ & 10 & 0.010 \\
\hline
\end{tabular}

Considering this data and the alternative hypothesis which states that participants in the EG would execute fewer context switches than those in the $\mathbf{C G}$ (i.e., $\mathbf{C G}>\mathbf{E G}$ ) overall, the results show that the participants in the EG did, in fact, execute significantly fewer context switches than those in the CG. These results support that the process was more streamlined for the EG. This outcome is directly in line with the results of the task time analysis performed previously.

5.2.6 Task 1 Results. In task T1, participants were asked to answer a set of questions to assess their interpretation of a Docker Compose configuration which was provided for this end. In this section, we analyzed the score results for these questions. The questions were split between two exercises: (1) considered true or false statements and (2) short answers in a specific format. For the answers in exercise 2, a strict all-or-nothing correction criterion was adopted in which an answer would only be considered as correct if it fully matched the expected solution and incorrect otherwise.

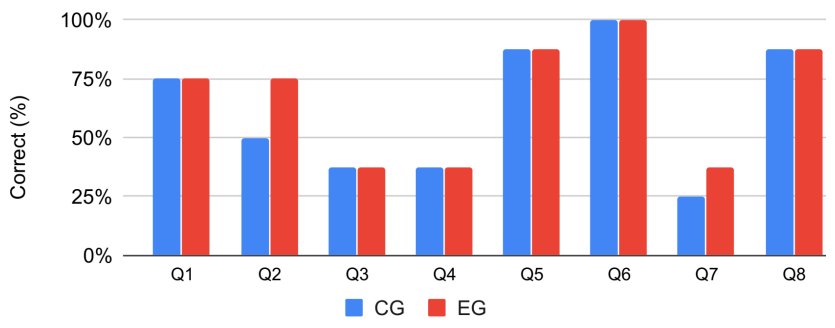

Figure 5: Percentage of participants that answered the questions of $\mathrm{T} 1$ correctly.

Fig. 5 displays the percentage of participants which answered correctly for each of the 8 questions considered in both exercises. We can see that there is only a minor difference between Q2 and Q7, in which more participants of the EG answered correctly.

Table 7: Summary of the results of the McNemar test applied to T1 (questions Q2 and Q7).

\begin{tabular}{|c|c|c|c|c|}
\hline & \multirow{2}{*}{$\frac{\text { CG }}{\%}$} & \multirow{2}{*}{$\frac{\text { EG }}{\%}$} & \multicolumn{2}{|c|}{ McNemar } \\
\hline & & & $H_{1}$ & $\rho$ \\
\hline Q2 & 50 & 75 & $>$ & 0.313 \\
\hline Q7 & 25 & 37.5 & $>$ & 0.500 \\
\hline
\end{tabular}

Table 7 summarizes the result of the McNemar test for Q2 and Q7. Considering this data and the expected alternative hypothesis which states that more participants in the EG would answer the questions correctly than those of the CG (CG < EG), the results don't demonstrate that significantly more participants of the EG answered questions correctly than those of the CG, for any of the questions. Taking into account these results, we can't conclude that the prototype was successful in increasing the correctness of answers. We believe that these results are partly attributed to the design of the questions. Some required a deeper knowledge of Docker Compose including some concepts which were not directly conveyed by prototype while others required a higher level of indirectness such as searching for the answer on the image documentation in Docker Hub. Despite this, it is still interesting to note that the results were similar across groups even though the participants in the EG relied far less on documentation resources. These results may suggest that the knowledge gained from the information conveyed by the prototype was comparable to that of reading documentation resources, at least for the concepts addressed in the task.

\subsection{Assessment Survey}

The contents of this survey differed between groups. They collected data about three perception-based metrics: Perceived Ease of Use (PEOU), Perceived Usefulness (PU), and Intention to Use (ITU). It featured a set of identical questions to evaluate possible environmental deviations and procedure understandably along with a set of similar questions to assess PEOU (except for 1 question to the EG since it did not apply to the CG). The survey provided to EG featured an additional set of questions to evaluate the perceived usefulness of individual features and overall PU as well as ITU sentiment towards the prototype. The questions which focused on PU were formulated to compare the usefulness of the prototype in relation to the participant's perception of the conventional method and toolchain.

Table 8: Summary of the answers to the common Likertscale questions in the assessment survey.

\begin{tabular}{|c|c|c|c|c|c|c|c|}
\hline & \multicolumn{2}{|c|}{$\mathrm{CG}$} & \multicolumn{2}{|c|}{$\mathrm{EG}$} & \multicolumn{3}{|c|}{ MW-U } \\
\hline & $\bar{x}$ & $\sigma$ & $\bar{x}$ & $\sigma$ & $H_{1}$ & $\mathrm{u}$ & $\rho$ \\
\hline ENV1 & 4.13 & 1.356 & 3.75 & 1.282 & $\neq$ & 25.5 & 0.231 \\
\hline ENV2 & 1.88 & 1.356 & 2.13 & 1.458 & $\neq$ & 29 & 0.367 \\
\hline
\end{tabular}

5.3.1 Environment. Analyzing the data in Table 8 and considering the alternative hypothesis which states that the perception of the CG of environment factors differs from the EG for all environmentrelated questions, the results demonstrate that there is not a significant difference between both groups. These results support the hypothesis that the influence of environmental factors on performance during tasks was balanced across both groups and therefore, did not have a meaningful impact on the results.

5.3.2 Perceived Process Understandability. It is essential to note the difference of the PPU between both groups. As shown by Table 9 , there is a significant difference regarding the task description across both groups, and the means suggest that the EG found the task descriptions more understandable than the CG. Although this discrepancy may imply a threat to validity, since it could entail that differences in performance are related to the difficulty in understanding the instructions, we argue that it may have been the 
Table 9: Summary of the results of the answers to the common Likert-scale questions in the assessment survey.

\begin{tabular}{lccccccccc} 
& \multicolumn{2}{c}{$\mathrm{CG}$} & & \multicolumn{2}{c}{$\mathrm{EG}$} & & \multicolumn{3}{c}{$\mathrm{MW}-\mathrm{U}$} \\
\cline { 2 - 3 } \cline { 7 - 8 } \cline { 6 - 8 } & $\bar{x}$ & $\sigma$ & & $\bar{x}$ & $\sigma$ & & $H_{1}$ & $\mathrm{u}$ & $\rho$ \\
\hline PPU1 & 2.25 & 1.282 & & 1.25 & 0.463 & & $\neq$ & 17.0 & 0.080 \\
PPU2 & 3.00 & 0.926 & & 1.50 & 0.756 & & $\neq$ & 6.5 & 0.005 \\
\hline PPU1. I found the procedure instructions complex and difficult to follow. \\
PPU2. I found the task descriptions complex and difficult to follow.
\end{tabular}

result of the more pronounced difficulties felt by the participants of the CG. They generally struggled more to complete tasks, which may have influenced their judgment. Another possibility is that the participants interpreted the questions wrong and mistook the intent of the statement with ease of implementation. In contrast, the prototype used by the participants in the EG provided a more streamlined and focused experience (as supported by the lower context switching) which helped participants to concentrate more clearly on the provided instructions and were as a result less fatigued by the end of the sessions.

Table 10: Summary of the results of the answers to the Likert-scale questions related to perceived ease of use (PEOU) in the assessment survey. $\left({ }^{*}\right)$ PEOU4 was exclusive for the EG.

\begin{tabular}{|c|c|c|c|c|c|c|c|}
\hline & \multicolumn{2}{|c|}{$\mathrm{CG}$} & \multicolumn{2}{|c|}{ EG } & \multicolumn{3}{|c|}{ MW-U } \\
\hline & $\bar{x}$ & $\sigma$ & $\bar{x}$ & $\sigma$ & $H_{1}$ & $\mathrm{u}$ & $\rho$ \\
\hline PEOU1 & 2.64 & 1.188 & 1.00 & 0.000 & $>$ & 8.0 & 0.002 \\
\hline PEOU2 & 2.63 & 0.916 & 1.13 & 0.354 & $>$ & 5.5 & 0.001 \\
\hline PEOU3 & 3.63 & 0.744 & 5.00 & 0.000 & $<$ & 4.0 & 0.001 \\
\hline PEOU4* & $\mathrm{n} / \mathrm{a}$ & $\mathrm{n} / \mathrm{a}$ & 4.88 & 0.354 & $\mathrm{n} / \mathrm{a}$ & $\mathrm{n} / \mathrm{a}$ & $\mathrm{n} / \mathrm{a}$ \\
\hline $\begin{array}{l}\text { PEOU1. O } \\
\text { PEOU2. I } \\
\text { PEOU3. I } \\
\text { PEOU4. }\end{array}$ & Ill, If & $\begin{array}{l}\text { to def } \\
\text { d the t }\end{array}$ & ficult & $\begin{array}{l}\text { the } \\
\text { n. }\end{array}$ & & & \\
\hline
\end{tabular}

5.3.3 Perceived Ease of Use. By analyzing the data in Table 10 and considering the hypothesis that participants in the EG would find the prototype easier to use (i.e., CG > EG for PEOU1 and PEOU2 and $\mathbf{C G}<\mathbf{E G}$ for PEOU3) for all equivalent PEOU questions, the results demonstrate that the EG did indeed find that it was significantly easier to work with the prototype. Therefore we can state with some confidence that participants did find the prototype easier to use than the conventional method. Additionally, the exclusive question PEOU4 also demonstrates that the participants in EG strongly agreed that the tool easy to learn.

5.3.4 Features. The following sections focus on the questions which were exclusive to the survey provided to the EG.

The survey provided to the EG contained a section dedicated to evaluating the perceived usefulness of individual features, namely, the visual map of artefacts (VM), Docker Hub integration (DHI), visual feedback (VF), and executing commands on the UI (UIC). The goal of these questions was to assess the perceived usefulness with a higher degree of granularity so that we could better understand the impact of each feature in the overall perception. Table 11 summarizes the obtained results.
Table 11: Summary of the results of the answers to the Likert-scale questions related to perceived usefulness of features in the assessment survey. N/A means not applicable.

\begin{tabular}{|c|c|c|c|c|c|c|c|c|}
\hline & \multicolumn{2}{|c|}{ VM } & \multicolumn{2}{|c|}{ DHI } & \multicolumn{2}{|c|}{ VF } & \multicolumn{2}{|c|}{ UIC } \\
\hline & $\bar{x}$ & $\sigma$ & $\bar{x}$ & $\sigma$ & $\bar{x}$ & $\sigma$ & $\overline{\bar{x}}$ & $\sigma$ \\
\hline ULE & 4.88 & 0.35 & 4 & 1.20 & 4.50 & 0.76 & $\mathrm{n} / \mathrm{a}$ & $\mathrm{n} / \mathrm{a}$ \\
\hline UQ & 4.75 & 0.46 & 4 & 1.20 & 4.75 & 0.71 & $\mathrm{n} / \mathrm{a}$ & $\mathrm{n} / \mathrm{a}$ \\
\hline DLE & 4.88 & 0.35 & 4 & 1.20 & $\mathrm{n} / \mathrm{a}$ & $\mathrm{n} / \mathrm{a}$ & 4.75 & 0.71 \\
\hline DQ & 4.88 & 0.35 & 4 & 1.20 & $\mathrm{n} / \mathrm{a}$ & $\mathrm{n} / \mathrm{a}$ & 4.75 & 0.71 \\
\hline
\end{tabular}

ULE. Understand stacks with less effort.

UQ. Understand stacks more quickly.

DLE. Define stacks with less effort.

DQ. Define stacks more quickly.

Table 12: Summary of the results of the answers to the Likert-scale questions related to the overall perceived usefulness in the assessment survey.

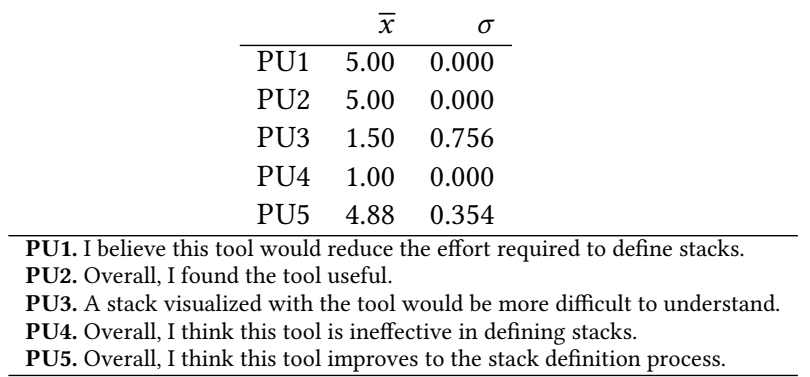

Considering the data displayed in Table 11, we can conclude that the feature considered most useful was the visual map of artefacts (VM) while the least was the Docker Hub integration (DHI). These results match our expectations as the DHI feature was secondary and mostly added for ease-of-use and convenience. The designed tasks did not take full advantage of this feature since participants were able to copy and paste the image names and tags from the provided script without the need to locate them manually. In contrast, the VM feature was the direct result of the hypothesis of this dissertation and corresponded to the most novel and premeditated feature. Regardless, the response was positive for all features.

5.3.5 Perceived Usefulness. Taking into the account the combined feedback of the usefulness of individual features (Table 11) and overall usefulness (Table 12), we can state that participants did indeed find the tool useful.

5.3.6 Intention to Use. Taking into account the results displayed in Table 13, we can state with some confidence that participants do indeed intend to use the tool in the future.

Overall, the results demonstrate that the response to the prototype was overwhelmingly positive and generally very consistent across participants. The participants found the approach more straightforward to use than the conventional method, generally useful, and were interested in using it in the future. 
Table 13: Summary of the results of the answers to the Likert scale questions related to the overall perceived usefulness in the assessment survey.

\begin{tabular}{lrr} 
& $\bar{x}$ & $\sigma$ \\
\hline ITU1 & 4.50 & 0.535 \\
ITU2 & 4.75 & 0.463 \\
ITU3 & 5.00 & 0.000 \\
ITU4 & 4.50 & 0.756 \\
ITU5 & 4.75 & 0.463
\end{tabular}

ITU1. This tool would make it easier for practitioners to define service stacks.

ITU2. Using this tool would make it easier to explain the stack.

ITU3. I would recommend this tool to work with Docker Compose.

ITU4. I would like to use this tool in the future.

ITU5. It would be easy for me to become skillful in using this tool.

\section{CONCLUSIONS}

Our findings support the hypothesis that a completely visual approach for orchestration does indeed generally reduce development time and error-proneness significantly. The most visible benefits were observed during implementation-based tasks (T3.1 and T3.2). We also conclude that regarding time-related improvements, the results suggest that the observed gains were mostly due to reductions in time spent on reading documentation. Additionally, the analysis of the context switches suggests that the experience was overall more streamlined when using the prototype.

The findings related to perception-based metrics, although less conclusive, were positive overall. The participants overwhelmingly felt that the tool was more comfortable to use, was generally useful, and had strong intentions of using it in the future. These results give us some confidence that developers find the tool easy-to-use and intuitive, considering the steps and actions needed to successfully configure some orchestration specifications. This overall developer experience in using such visual programming approach for orchestration, along with the use of similar approaches in other application domains, make us believe that such tools can play an important role in software development tasks, at least as a complement to the commonly used tools.

While the obtained results are promising, the prototype is not production-ready, and additional research would be useful to further consolidate and confirm our findings with more confidence, primarily to address some identified validation threats, namely: (1) psychological bias-i.e., it was somewhat evident for the participants to identify in which EG they were, (2) scarce sample size-i.e., there is the need to re-run the experiment with larger sample size, (3) sample bias, since all the participants were students with similar backgrounds, (4) environment influences, since the experiments were run remotely we cannot measure the influence of uncontrolled external factors, and (5) auto-layout inefficiencies which could have impacted the subject's capabilities to understand the underlying orchestration specification. Nonetheless, the solution is ready-available and the experiments reproducible ${ }^{5}$.

\section{ACKNOWLEDGMENTS}

Thank you to David Reis and Jessica Diaz for discussing earlier versions of this work with us. This work was partially funded by the Integrated Masters in Informatics and Computing Engineering of the Faculty of Engineering, University of Porto (FEUP) and the Portuguese Foundation for Science and Technology (FCT), ref. SFRH/BD/144612/2019.

\section{REFERENCES}

[1] Nuha Alshuqayran, Nour Ali, and Roger Evans. 2016. A systematic mapping study in microservice architecture. 2016 IEEE 9th International Conference on Service-Oriented Computing and Applications, SOCA 2016 (2016), 44-51.

[2] Danilo Ardagna, Elisabetta Di Nitto, Parastoo Mohagheghi, Sébastien Mosser, Cyril Ballagny, Francesco D'Andria, Giuliano Casale, Peter Matthews, Cosmin Septimiu Nechifor, Dana Petcu, Anke Gericke, and Craig Sheridan. 2012. MODAClouds: A model-driven approach for the design and execution of applications on multiple clouds. 2012 4th International Workshop on Modeling in Software Engineering, MiSE 2012 - Proceedings (2012), 50-56.

[3] Alexander Bergmayr, Javier Troya, Patrick Neubauer, Manuel Wimmer, and Gerti Kappel. 2014. UML-based cloud application modeling with libraries, profiles, and templates. CEUR Workshop Proceedings 1242, 317859 (2014), 56-65.

[4] J. P. Dias, J. P. Faria, and H. S. Ferreira. 2018. A Reactive and Model-Based Approach for Developing Internet-of-Things Systems. In 2018 11th International Conference on the Quality of Information and Communications Technology (QUATIC). $276-281$.

[5] Jezz Humble and David Farley. 2010. Continuous Delivery: Reliable Software Releases through Build, Test, and Deployment Automation. Pearson Education. 497 pages. https://doi.org/10.1007/s13398-014-0173-7.2 arXiv:arXiv:1011.1669v3

[6] Ann Mary Joy. 2015. Performance comparison between Linux containers and virtual machines. Conference Proceeding - 2015 International Conference on Advances in Computer Engineering and Applications, ICACEA 2015 (2015), 342-346.

[7] Pedro Lourenço, João Pedro Dias, Ademar Aguiar, and Hugo Sereno Ferreira. 2019. Cloudcity: A live environment for the management of cloud infrastructures. ENASE 2019 - Proceedings of the 14th International Conference on Evaluation of Novel Approaches to Software Engineering (2019), 27-36.

[8] Pedro Lourenço, João Pedro Dias, Ademar Aguiar, Hugo Sereno Ferreira, and André Restivo. 2020. Experimenting with Liveness in Cloud Infrastructure Management. In Evaluation of Novel Approaches to Software Engineering, Ernesto Damiani, George Spanoudakis, and Leszek A. Maciaszek (Eds.). Springer International Publishing, Cham, 58-82.

[9] Russ McKendrick and Scott Gallagher. 2017. Mastering Docker-Second Edition. Packt Publishing. 384 pages.

[10] Claus Pahl, Antonio Brogi, Jacopo Soldani, and Pooyan Jamshidi. 2017. Cloud Container Technologies: a State-of-the-Art Review. IEEE Transactions on Cloud Computing 7161, c (2017), 1-14. https://doi.org/10.1109/TCC.2017.2702586

[11] Sudhir Pandey. 2012. Investigating community, reliability and usability of cfengine, chef and puppet. Master's thesis.

[12] Fawaz Paraiso, Stéphanie Challita, Yahya Al-Dhuraibi, and Philippe Merle. 2017. Model-driven management of docker containers. IEEE International Conference on Cloud Computing, CLOUD (2017), 718-725.

[13] Akond Rahman, North Carolina, Chris Parnin, North Carolina, Laurie Williams, and North Carolina. [n.d.]. Gang of Eight : A Defect Taxonomy for Infrastructure as Code Scripts. ([n. d.]). Accepted submission for the International Conference on Software Engineering (ICSE) 2020.

[14] Julio Sandobalin, Emilio Insfran, and Silvia Abrahao. 2018. ARGON: A Tool for Modeling Cloud Resources. Lecture Notes in Computer Science (including subseries Lecture Notes in Artificial Intelligence and Lecture Notes in Bioinformatics) 10797 LNCS, November (2018), 393-397. https://doi.org/10.1007/978-3-319-91764-1_37

[15] Julio Sandobalin, Emilio Insfran, and Silvia Abrahao. 2020. On the Effectiveness of Tools to Support Infrastructure as Code : Model-Driven versus Code-Centric. IEEE Access 8 (2020).

[16] Tiago Boldt Sousa, Ademar Aguiar, Hugo Sereno Ferreira, and Filipe Figueiredo Correia. 2016. Engineering software for the cloud: patterns and sequences. In Proceedings of the 11th Latin-American Conference on Pattern Languages of Programming. 1-8.

[17] Tiago Boldt Sousa, Filipe Figueiredo Correia, and Hugo Sereno Ferreira. 2015. Patterns for Software Orchestration on the Cloud. In Proceedings of the 22nd Conference on Pattern Languages of Programs (Pittsburgh, Pennsylvania) (PLoP '15). The Hillside Group, USA, Article 17, 12 pages.

[18] Tiago Boldt Sousa, Hugo Sereno Ferreira, and Filipe Figueiredo Correia. 2018. Overview of a Pattern Language for Engineering Software for the Cloud. In Proceedings of the 25th Conference on Pattern Languages of Programs (PLoP '18). The Hillside Group, USA, 1-9.

[19] Michael Tiegelkamp and Karl-Heinz John. 1995. IEC 61131-3: Programming industrial automation systems. Vol. 14. Springer.

[20] Denis Weerasiri, Moshe Chai Barukh, Boualem Benatallah, and Cao Jian. 2016. CloudMap: A Visual Notation for Representing and Managing Cloud Resources. 28th International Conference, CAiSE 2016 (2016), 427-443. 